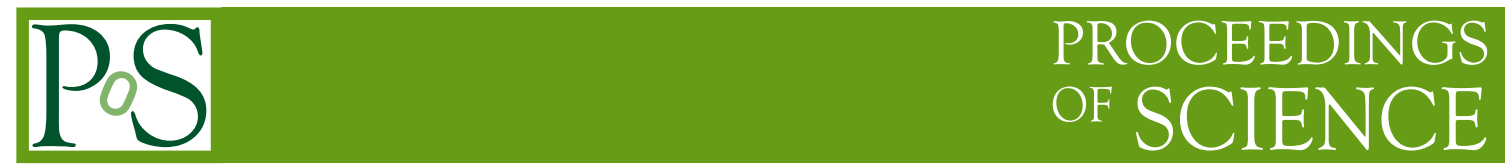

\title{
Strange-baryon spectroscopy with HADES
}

\author{
Kirill Lapidus* ${ }^{\dagger}$ \\ Excellence Cluster "Universe" \\ TU Munich \\ E-mail: kirill.lapidus@ph.tum.de
}

We discuss an analysis of the strange baryon resonances $\Sigma(1385)^{+}$and $\Lambda(1405)$ measured with the High-Acceptance Di-Electron Spectrometer (HADES) in proton-proton collisions at $3.5 \mathrm{GeV}$ beam kinetic energy. A strategy for the signal extraction is outlined and preliminary results are presented.

XLIX International Winter Meeting on Nuclear Physics

24-28 January 2011

BORMIO, Italy

\footnotetext{
* Speaker.

${ }^{\dagger}$ Talk given on behalf of the HADES collaboration
} 


\section{Introduction}

We study two strange baryon resonances close to the antikaon-nucleon threshold, $\Sigma(1385)$ and $\Lambda(1405)$. Whereas the $\Sigma(1385)$ resonance is well understood as a usual 3-quark state, the genesis of the $\Lambda(1405)$ is a subject of an active discussion. The mass of this resonance is puzzling by itself: though $\Lambda(1405)$ contains a strange valence quark, it is the lightest negative-parity baryon (the lightest non-strange negative-parity baryon is $\left.N^{*}(1520)\right)$. Moreover, the large mass difference between $\Lambda(1405)$ and $\Lambda(1520)$ calls for a large spin-orbit force, but such a strong splitting is not observed for other baryons.

Another evidence for the non-trivial nature of the $\Lambda(1405)$ comes from lattice QCD calculations. While for most baryons the lattice evaluations can predict masses with a $10 \%$ accuracy [1], for the $\Lambda(1405)$ the predicted mass is $\sim 300 \mathrm{MeV} / c^{2}$ higher than the measured value, which supports a molecular or a pentaquark hypothesis for the $\Lambda(1405)[1,2]$. As further speculated in [3], lattice results indicate that the $\Lambda(1405)$ could be a mixed $3 q-5 q$ state.

In recent coupled-channel calculations based on the chiral dynamics, the $\Lambda(1405)$ is understood as a dynamically generated resonance that couples to the $\Sigma \pi$ and $\bar{K} N$ channels [6, 7]. Because of the coupling to the different channels and the interference between the isospin 0 and 1 components, the observed properties of the $\Lambda(1405)$ are expected to depend on both the production reaction and the decay mode. For example, as predicted in [5], a reaction $K^{-} d \rightarrow \pi \Sigma n$ favors a formation of the $\Lambda(1405)$ resonance, which couples mostly to the $\bar{K} N$ state, therefore the calculated lineshape of the $\Lambda(1405)$ reveals a position of the pole at $1420 \mathrm{MeV}$. From the experimental side, the CLAS collaboration performed a measurement of the $\gamma+p \rightarrow K^{+}+\Lambda(1405)$ reaction at photon energies of up to $3.84 \mathrm{GeV}$ and reported an observation of different $\Lambda(1405)$ line shapes for the different decay channels (charged channels $\Sigma^{ \pm} \pi^{\mp}$ and a neutral decay channel $\Sigma^{0} \pi^{0}$ ).

While a number of experiments investigated the $\Lambda(1405)$ production in pion-proton and antikaon-proton reactions, proton-proton reactions did not receive an adequate attention. Recently, the $\Lambda(1405)$ was measured in the $\Sigma^{0} \pi^{0}$ decay channel in proton-proton collisions at the $3.65 \mathrm{GeV} / \mathrm{c}$ beam momentum with the ANKE spectrometer at COSY [8]. The obtained spectral shape exhibit a prominent structure around $1400 \mathrm{MeV} / c^{2}$ and was found to be consistent with the results of bubble-chamber data taken in $\pi^{-} p \rightarrow K^{0} \Lambda(1405)$ and $K^{-} p \rightarrow \Lambda(1405) \pi^{+} \pi^{-}$reactions.

Another aspect of the rich $\Lambda(1405)$ physics is related to the formation of deeply bound antikaonnuclear states, predicted in [9]. In [10] it was speculated that the $\Lambda(1405)$, assumed to be mainly a $\bar{K} N$ bound state, can act as a building block, or a "doorway" for the formation of a $K^{-} p p$ systemthe lightest possible bound kaonic state ${ }^{1}$.

We aim to enrich the scarce data on the $\Lambda(1405)$ resonance production in proton-proton collisions; a measurement of the $\Sigma(1385)$ provides an important benchmark for the analysis procedure.

\section{The HADES experiment}

The High-Acceptance Di-Electron Spectrometer (HADES) is a modern multi-purpose detector currently operating at the SIS18 heavy-ion synchrotron (GSI Helmholtzzentrum, Darmstadt) in the region of kinetic beam energies of 1-3 AGeV. The main components of the experimental set-up

\footnotetext{
${ }^{1}$ Recently, a new, much lighter $\bar{K} N \pi$ quasibound state was predicted in [12].
} 
are a superconducting magnet, four planes of Multiwire Drift Chambers used for the tracking of charged particles, a Time-of-Flight wall and a hadron blind RICH detector, cf. [13] for details.

In 2007 a measurement of proton-proton collisions at the beam kinetic energy of $3.5 \mathrm{GeV}$ was performed. The beam with an average intensity $\sim 1 \cdot 10^{6}$ particles/s was incident on a liquid hydrogen target with a columnar density $0.35 \mathrm{~g} / \mathrm{cm}^{2}$ and the total interaction probability of $\sim 2 \%$. In total, $1.2 \cdot 10^{9}$ events were collected.

\section{Analysis of the strange-baryon production in $p+p$ collisions}

\section{$3.1 \Sigma(1385)^{+}$}

We performed an exclusive measurement of the $\Sigma(1385)^{+}$in the following production and decay chain:

$$
p+p \rightarrow n+\Sigma(1385)^{+}+K^{+}, \Sigma(1385)^{+} \rightarrow \Lambda \pi^{+}, \Lambda \rightarrow p+\pi^{-} .
$$

As a first step of the data reduction, four charged particles $\left(p, K^{+}, \pi^{-}\right.$and $\left.\pi^{+}\right)$were identified with the help of measured energy losses in the sub-detectors of the HADES set-up. Afterwards, after selecting $\Lambda$ candidates, the missing mass of these four particles was constructed (see Fig. 1). A clear peak corresponding to the missed neutron is visible on top of the background that mainly originates from the misidentification of pions and protons as kaons. To model this background an elaborated side-band technique was developed [14]. With the fit to the missing mass spectra the contribution from the misidentification background is fixed.

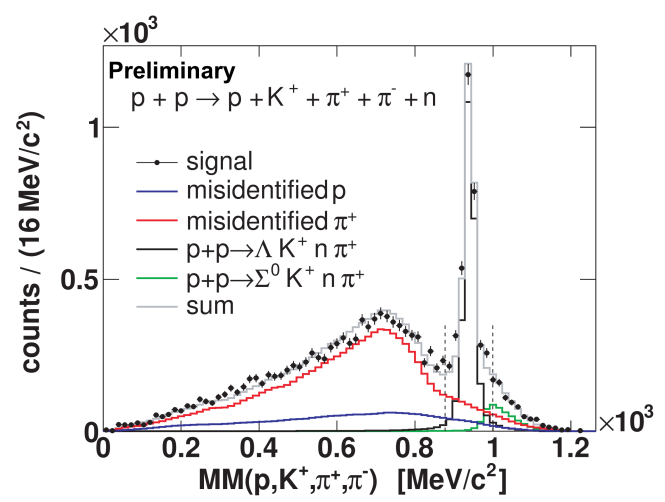

Figure 1: Missing mass of $p K^{+} \pi^{+} \pi^{-}$. Vertical dashed lines indicate the region used for the neutron selection

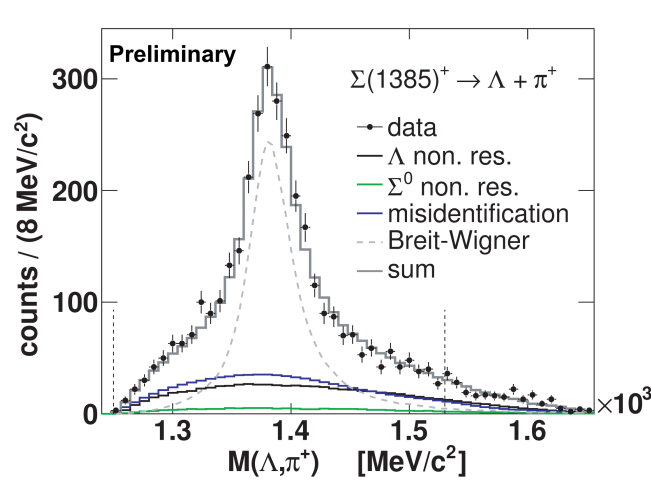

Figure 2: Invariant mass distribution of $\Lambda \pi^{+}$pairs. The dashed curve corresponds to the extracted $\Sigma(1385)^{+}$signal

Afterwards, a cut on the missed neutron is imposed, selecting events with the final state as expected from the reaction 3.1. In order to improve the precision of the measurement, a kinematical refit is applied, taking the neutron mass as a constraint. Then we construct the $\Lambda \pi^{+}$invariant mass spectrum (see Fig. 2). It reveals a resonant structure corresponding to the $\Sigma(1385)$ and a broad background. The main components of the background are wrong kaon identification and a reaction channel with the non-resonant production of the same final state particles as in the reaction 3.1 $\left(p+p \rightarrow n+\Lambda+\pi^{+}+K^{+}\right)$. 
After a simultaneous fit of the contributions from the physical channels to the data, we obtain a $\Sigma(1385)^{+}$signal, which is described by a relativistic Breit-Wigner distribution with the following parameters: $M_{\Sigma(1385)^{+}}=1382.99 \pm 0.79, \Gamma_{\Sigma(1385)^{+}}=39.20 \pm 2.04$ (quoted are statistical uncertainties only). The extracted values are in a good agreement with previous measurements and the analysis procedure is, therefore, justified.

The high statistics obtained $\left(\sim 1700\right.$ counts) allows to investigate the $\Sigma(1385)^{+}$production in more details. In particular, our on-going analysis of the angular distributions may deliver valuable information.

\section{$3.2 \Lambda(1405)$}

\subsubsection{Neutral decay}

The neutral decay mode $\Lambda(1405) \rightarrow \Sigma^{0} \pi^{0}$ is of particular interest, since the $\Sigma(1385)$ does not decay to this final state due to isospin conservation and, therefore, does not contaminate the $\Lambda(1405)$ signal.

Here, the following reaction chain was studied:

$$
p+p \rightarrow p+\Lambda(1405)+K^{+}, \Lambda(1405) \rightarrow \Sigma^{0} \pi^{0}, \Sigma^{0} \rightarrow \Lambda+\gamma, \Lambda \rightarrow p+\pi^{-} .
$$

Four charged particles are identified $\left(p, p, K^{+}\right.$and $\left.\pi^{-}\right)$. A $p K^{+}$missed mass spectrum is shown in Fig. 3. Clear peaks from the $\Lambda(1116), \Sigma(1190)$ and an overlapping $\Sigma(1385) / \Lambda(1405)$ complex are visible.

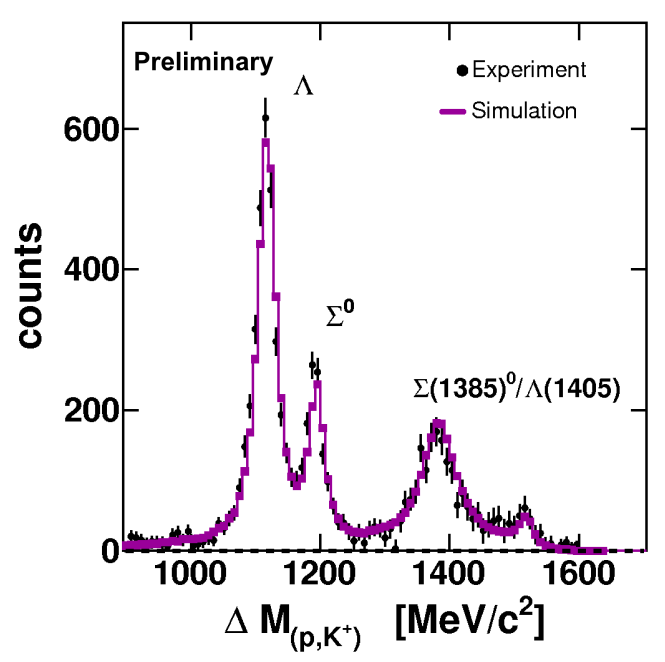

Figure 3: Missing mass distribution of $\mathrm{pK}^{+}$-pairs. Experimental data are shown as black circles, a magenta line corresponds to the sum of the simulated channels (see explanation in the text)

Since a number of physical channels contribute to the selected data sample, their contributions should be subtracted in order to obtain a pure $\Lambda(1405)$ signal. The following reaction channels were included in the simulation: $\Lambda K^{+} p, \Lambda K^{+} p \pi^{0}, \Lambda K^{+} p \pi^{-} \pi^{+}, \Sigma^{+} K^{+} p \pi^{-}, \Sigma^{+} K^{+} p \pi^{-} \pi^{0}, \Sigma^{0} K^{+} p \pi^{0}$, $\Sigma^{0} K^{+} p \pi^{-} \pi^{+}, \Sigma^{0} p K^{+}, \Lambda(1405) p K^{+}, \Lambda(1520) p K^{+}$. With a simultaneous fit of all contributing channels to the experimental data we aim at extracting their strengths. 


\subsubsection{Charged decays}

We have analyzed charged decays of the $\Lambda(1405)$ in the following reactions:

$$
p+p \rightarrow p+\Lambda(1405)+K^{+}, \Lambda(1405) \rightarrow \Sigma^{ \pm} \pi^{\mp}, \Sigma^{ \pm} \rightarrow n+\pi^{ \pm} .
$$

As in the case of the $\Sigma(1385)^{+}$analysis, four charged particles $\left(p, \pi^{+}, \pi^{-}, K^{+}\right)$are reconstructed in the final state. After extracting the neutron via mixing mass technique, we construct the missed mass spectra of the $p K^{+} \pi^{\mp}$ (see Fig. 4). The spectra show narrow signals of the $\Sigma^{+}(1189)$ and $\Sigma^{-}(1197)$ resonances. A cut on the missed mass spectra is imposed, selecting the $\Sigma$ resonance. After this cut, missing mass spectra of $p K^{+}$(see Fig. 5) contain the following contributions: $\Sigma(1385)^{0}, \Lambda(1405), \Lambda(1520)$, the (known) kaon misidentification background and the non-resonant production of the $\Sigma^{ \pm}+\pi+K^{+}$state, which was simulated according to the phase space model [15]. An important feature of the obtained data is that in order to reproduce the missing mass spectra (Fig. 5) the $\Lambda(1405)$ resonance with a rather low Breit-Wigner peak mass of $1385 \mathrm{MeV} / \mathrm{c}^{2}$ had to be simulated. Preliminary results suggest that about $600 \Lambda(1405)$ resonances can be reconstructed in both charged decay channels.
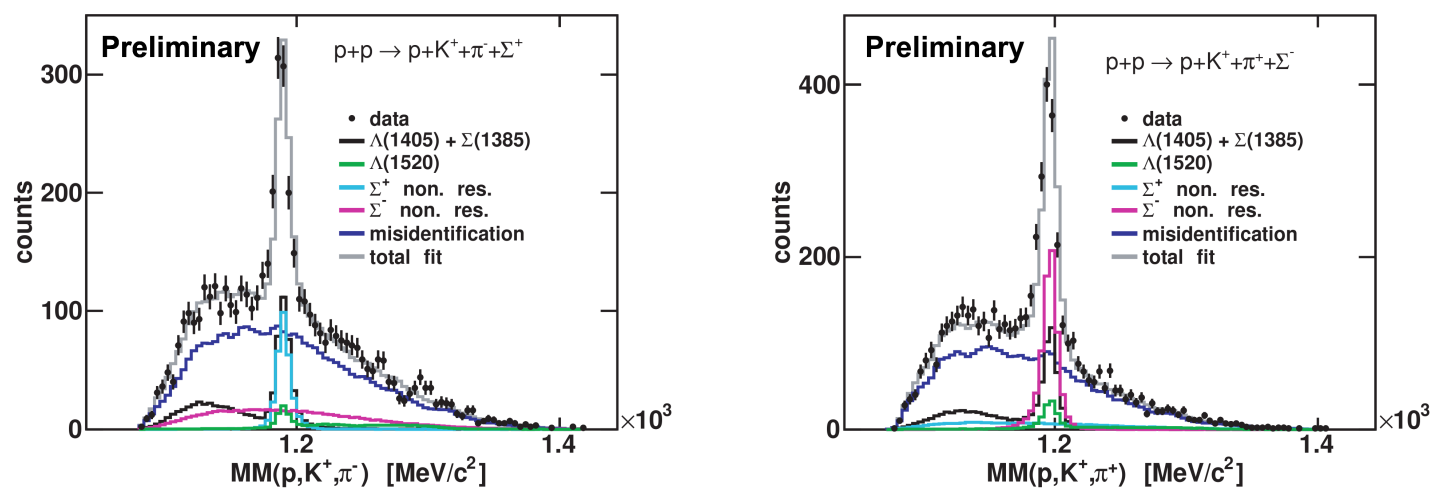

Figure 4: Missing mass spectra of $p K^{+} \pi^{-}$(left) and $p K^{+} \pi^{+}$(right). Experimental data are shown as black circles, colored histograms correspond to the different contributing channels
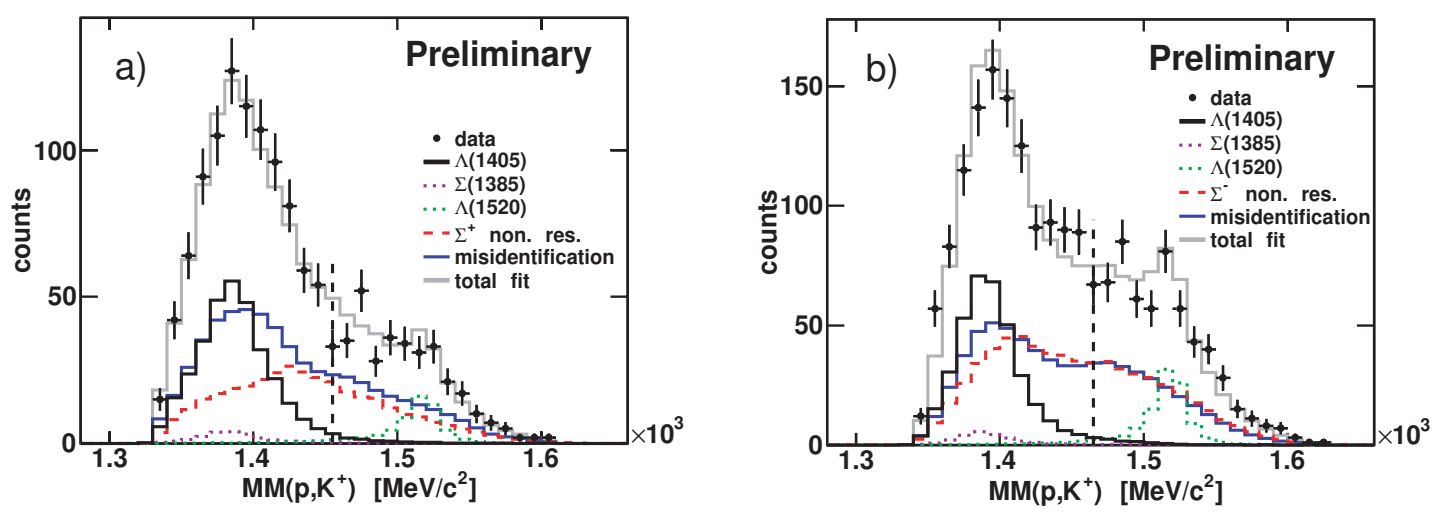

Figure 5: Missing mass distribution of the $p K^{+}$pairs, after the $\Sigma^{+}$(left) and $\Sigma^{-}$(right) selection cut. Experimental data are shown with black circles, colored histograms correspond to the different contributing channels 


\section{Summary}

In this contribution we reported on the on-going analysis of the $\Sigma(1385)^{+}$and $\Lambda(1405)$ production in proton-proton collisions at $3.5 \mathrm{GeV}$ beam kinetic energy. A reconstruction of the $\Sigma(1385)^{+}$signal delivers an important test of the analysis procedure and - thanks to significant statisticsallows a detailed study of this resonance. For the $\Lambda(1405)$, we aim to obtain its lineshape in the three decay channels. Preliminary results delivered by the charged decay mode analysis indicate that the Breit-Wigner peak mass of the $\Lambda(1405)$ is located below $1400 \mathrm{MeV} / \mathrm{c}^{2}$.

In future, a planned pion-beam program opens an opportunity to the HADES experiment to perform a measurement of the $\Lambda(1405)$ production in pion-proton and pion-nucleus reactions. The foreseen upgrade of the HADES setup with an electromagnetic calorimeter will allow, for the first time, for an exclusive reconstruction of the $\Lambda(1405)$ resonance in pion-induced reactions in the neutral decay mode.

\section{Acknowledgments}

The HADES collaboration gratefully acknowledges the support by the BMBF through grants 06MT9156, 06GI146I, 06FY171, 06FY91001, and 06DR9059D (Germany), by GSI (TKrue 1012, GI/ME3, OF/STR), by the Helmholtz Alliance HA216/EMMI, by the Excellence Cluster Universe (Germany), by grants GA AS CR IAA100480803 and MSMT LC 07050MSMT (Czech Republic), by grant KBN5P03B 14020 (Poland), by INFN (Italy), by CNRS/IN2P3 (France), by grants MCYT FPA2000-2041-C02-02 and XUGA PGID FPA2009-12931 T02PXIC20605PN (Spain), by grant UCY- 10.3.11.12 (Cyprus), by INTAS grant 06-1000012-8861 and EU contract RII3-CT506078 .

\section{References}

[1] Y. Nemoto, N. Nakajima, H. Matsufuru and H. Suganuma, Phys. Rev. D 68 (2003) 094505.

[2] T. T. Takahashi and M. Oka, Prog. Theor. Phys. Suppl. 186 (2010) 172.

[3] N. Ishii, T. Doi, M. Oka and H. Suganuma, Prog. Theor. Phys. Suppl. 168 (2007) 598.

[4] L. S. Kisslinger and E. M. Henley, arXiv:0911.1179 [hep-ph].

[5] D. Jido, E. Oset and T. Sekihara, arXiv:1008.4423 [nucl-th].

[6] T. Hyodo, W. Weise, D. Jido, L. Roca and A. Hosaka, Mod. Phys. Lett. A 23 (2008) 2393.

[7] D. Jido, J. A. Oller, E. Oset, A. Ramos and U. G. Meissner, Nucl. Phys. A 725 (2003) 181.

[8] I. Zychor et al., Phys. Lett. B 660 (2008) 167.

[9] Y. Akaishi and T. Yamazaki, Phys. Rev. C 65 (2002) 044005.

[10] T. Yamazaki and Y. Akaishi, Phys. Lett. B 535 (2002) 70.

[11] T. Yamazaki and Y. Akaishi, Phys. Rev. C 76 (2007) 045201.

[12] A. Gal and H. Garcilazo, arXiv:1103.4757 [nucl-th].

[13] G. Agakishiev et al. [HADES Collaboration], Eur. Phys. J. A 41 (2009) 243. 
[14] J. Siebenson, Diploma Thesis, Technische Universität München (2010).

[15] J. Siebenson, L. Fabbietti, A. Schmah and E. Epple [HADES Collaboration], PoS BORMIO2010 (2010) 052. 